\title{
Effects of Repeated Sprint Training in Hypoxia on Physical Performance Among Athletes: A Systematic Review
}

\author{
Efectos del Entrenamiento de Velocidad Repetida en Hipoxia Sobre \\ el Rendimiento Físico Entre Atletas: Una Revisión Sistemática
}

Milan Zelenovic ${ }^{1}$; Titta Kontro²; Tijana Stojanovic ${ }^{3}$; Dan Iulian Alexe ${ }^{4}$; Danijel Bozic ${ }^{1}$; Nikola Aksovic $^{3}$; Bojan Bjelica ${ }^{1}$; Zoran Milanovic ${ }^{3}$ \& Sava Mihai Adrian ${ }^{4}$

ZELENOVIC, M.; KONTRO, T.; STOJANOVIC, T.; ALEXE, D. I.; BOZIC, D.; AKSOVIC, N.; BJELICA, B.; MILANOVIC, Z. \& ADRIAN, S. M. Effects of repeated sprint training in hypoxia on physical performance among athletes: A systematic review. Int. J. Morphol., 39(6):1625-1634, 2021.

SUMMARY: Repeated sprint training in hypoxia (RSH) represents an innovative method in the process of development and improvement of physical performance among athletes. However, there is less scientific data on this topic. The purpose of this systematic review was to investigate the effect of RSH method on motor abilities and performance among athletes, obtain new information, and expand the already known conclusions. The data search was performed of 4 electronic databases for the years 2000-2021 May as follows: Google Scholar, PubMed, Web of Science, and ResearchGate. This search with English language restriction was made by using the following terms, individually/combination: "repeated sprint ability", "hypoxia", "effects", "physical performance", "VO " 844 studies were indentified, and 14 studies were selected (11 male studies, 1 female study, 2 both sexes). Results of this systematic review, a total sample size of 347 athletes (40 females and 307 males, aged $15.3 \pm 0.5-35 \pm 7$ years), showed that RSH was an effective training method in improving all monitored variables (i.e. RSAmax, $\mathrm{VO}_{2 \max }$ ). However, it should be noted that major improvements were observed mainly in repeated sprint ability (RSA) tests, and less in aerobic tests (i.e. Wingate and Yo-Yo). In conclusion, based on current scientific studies, RSH is more effective method to improve the physical performance among athletes compared to repeated sprint training in normoxia (RSN). This study suggested that the RSH has a positive effect on the monitored variables in physical performance tests especially related to RSA.

KEY WORDS: Repeated sprint ability; Hypoxia; Effects; Physical performance; $\mathrm{VO}_{2 \max }$.

\section{INTRODUCTION}

Hypoxiais a physiological state of the body in which there is reduced oxygen saturation in body tissues, caused either by environmental factors (increase in altitude) or by internal factors (Pesta et al., 2011; Dekerle et al., 2012; Billaut et al., 2013; Galvin et al., 2013; Rupp et al., 2013). Training in conditions of hypoxia, most often in a natural environment (such as training at higher altitudes), or in artificial conditions (hyperbaric chambers, portable oxygen filtering devices), can be one of the ways to further improve sports performance (Girard et al., 2013).

Starting from the fact, that short-term sprints associated with short recovery periods, are the most common in all sports (Spencer et al., 2005), where the tendency to achieve the best results during continuous sprints, separated by short periods of active or passive rest
( $\leq 60 \mathrm{~s}$ ), is the repeated sprint ability (RSA), which is one of the most important part of physical performance among every athlete (Bishop et al., 2011). Given the high demands placed on athletes with the training method, which emphasizes the development of the ability to repeat highintensity efforts, can be offered a great benefit to athlete in competitions (Buchheit et al., 2010; Serpiello et al., 2012). Encouraged by such demands, and all the improvements to which high-intensity training methods contribute, athletes have modified and updated their training into repeated short-term sprint training in conditions of hypoxia, which are accompanied by incomplete recovery (repeated sprint training in hypoxia, RSH), and popularity and frequency of RHS method is increasing (Brocherie et al., 2017). During this type of training, RSH, there is a longer engagement and maintenance of the maximum number of

\footnotetext{
${ }^{1}$ Faculty of physical education and sport, University of East Sarajevo, East Sarajevo, Bosnia and Herzegovina.

${ }^{2}$ Faculty of Sport and Health Sciences, University of Jyväskylä, Jyväskylä, Finland.

${ }^{3}$ Faculty of sports and physical education, University of Nis, Nis, Republic of Serbia.

${ }^{4}$ Faculty of Movement, Sports and Health Sciences, Vasile Alecsandri University of Bacau, bacau, Romania.
} 
fast muscle fibers, which affects the appearance of better fatigue resistance in tests for the assessment of RSA (Brocherie et al., 2015a). This resistance occurs as a consequence of earlier, long-term mechanical work during training in conditions corresponding to higher altitudes, i.e. up to $3800 \mathrm{~m}$ (Brocherie et al., 2015a).

This type of training, RSH, is one of less studied areas in sports science, although the first scientific publication on training in conditions of hypoxia was published more than 50 years ago. Millet et al. (2019), found in their meta-analysis that RSH positively contributed to the improvement of physical performance, especially in RSA, among both individual and team sports athletes. It was also concluded that there was an increasing need for further investigation related to $\mathrm{RSH}$ as a training method and its underlying mechanism (Millet et al.). Brocherie et al. (2017), observed in their meta-analysis that there were the positive effects of RSH on the physical performance among athletes, especially on the ability to perform repetitive sprints. Furthermore, it was found the positive effect of this type of training in the field of endurance sports $\left(\mathrm{VO}_{2 \max }\right.$ was higher) (Brocherie et al., 2017). However, there is still less scientific data on the effect of RSH on individual physical performance among athletes, such as values of the different modalities of RSA, $\mathrm{VO}_{2 \max }$, as well as on performance in Wingate, and Yo-Yo (YYIR1/ YYIR2) tests.

The purpose of this systematic review was to investigate the effect of RSH training method on motor and functional abilities and performance among athletes, obtain new information, and expand the already known conclusions. Also, based on current scientific studies the effect of $\mathrm{RSH}$ on RSA, $\mathrm{VO}_{2 \max }$, performance in Wingate, and Yo-Yo (YYIR1/ YYIR2) tests was examined.

\section{MATERIAL AND METHOD}

This systematic review was conducted in accordance with the PRISMA (Preferred Reporting Items for Systematic Reviews and Meta-analyses) guidelines (Moher et al., 2009).

Search strategy and study selection. The data search was performed of 4 electronic databases for the years 2000-2021 May as follows: Google Scholar, PubMed, Web of Science, and Research Gate. This search with English language restriction was made by using the following terms and
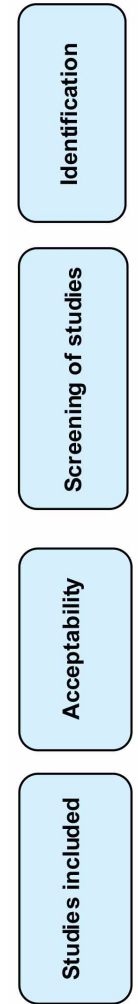

operators AND/OR individually/combination: "repeated sprint ability", "hypoxia", "effects", "physical performance", " $\mathrm{VO}_{2 \max }$ ". English was used as a language restriction, the relevance of the titles and abstracts of the identified studies was checked, and only the free full-text versions of original scientific studies in electronically available Journals were accepted for further analysis. The study selection was made by using inclusion and exclusion criteria. Furthermore, the reference lists of relevant study reports were searched to obtain more studies related to this research topic.

Literature search, identification and review of studies was conducted by five authors (MZ, TK, TS, DIA, DB), and the quality of assessment and data extraction was conducted independently by three authors (NA, BB, SMA). To identify relevant studies, all the titles were initially reviewed by the authors during electronic searches to exclude titles that were not relevant. In the initial review 844 potentially acceptable study reports were identified. The study selection process is shown in Figure 1. The fulltext versions of the selected studies, which met the inclusion criteria, were included in the procedure and reviewed by all authors, to make the final decision on inclusion in the systematic review of the research. Disagreements between authors were resolved by consensus or arbitration by ZM.

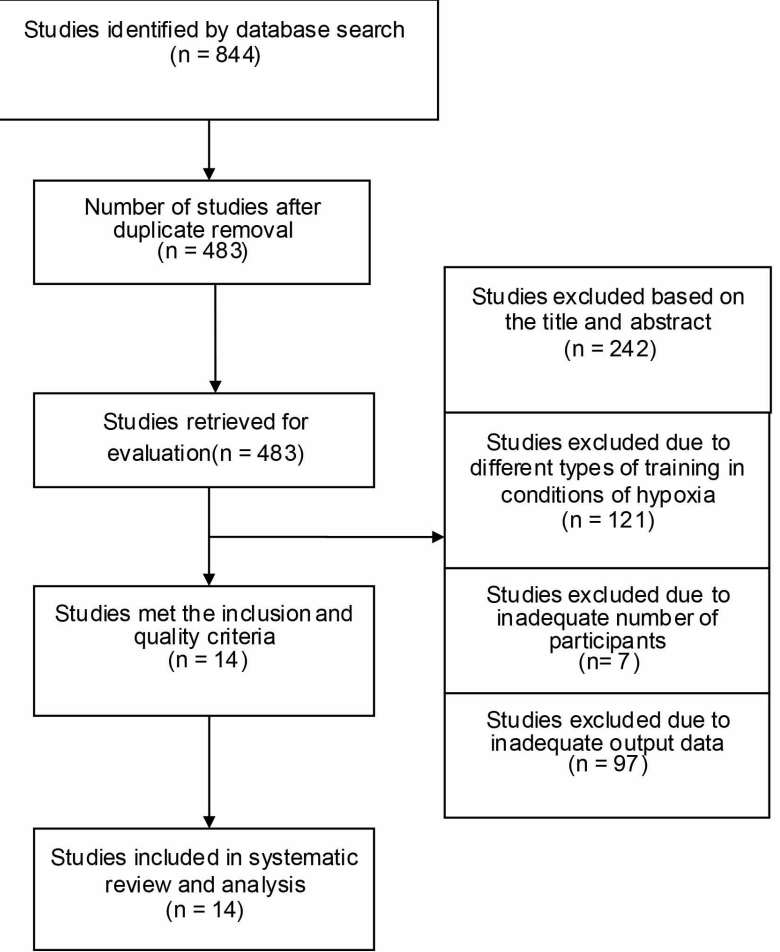

Fig. 1. Selection of studies for systematic review. 
Inclusion criteria. For the selection of the studies, which were included in the final analysis, the following criteria for inclusion were defined: (1) original scientific study reports; (2) studies based on longitudinal or parallel or cross-over design; (3) studies written in English; (4) sample of participants - active athletes; (5) duration of experimental treatments of at least 12 days; (6) experimental treatment under conditions of hypoxia and normoxia; (7) a minimum of two groups of participants ( 1 experimental - 1 control or 2 or more experimental); (8) primary dependent variables RSA (repeated sprint ability), $\mathrm{VO}_{2 \max }$ (maximum oxygen consumption), YYIR test, Mean and peak power (produced power during the test); (9) studies where only athletes from dry land sports were tested.

Exclusion criteria. Based on the following criteria, the studies were excluded from the further analysis: (1) studies based on cross-sectional design; (2) written studies in other languages; (3) inadequate sample of participants (nonathletes, obese, over 45 years of age, etc.); (4) studies with a lack of a control group or other experimental group; (5) the experimental treatment lasted less than 12 days; (6) studies where the experimental treatment was not realized in the conditions of hypoxia and normoxia; (7) studies in which the results were not adequately presented or the parameters required for further analysis are missing; (8) studies where the tests were based on animals; (9) studies where water sports athletes were tested, (10) studies related to the case study.

Data extraction. The selected studies in this systematic review are shown in Table I. For each study the parameters are shown: (1) characteristics of the study, including author (s) and year of publication, (2) study design, (3) information on participants such as sample size, sex, age, number of groups and sports groups/type, (4) a description of the experimental procedure that includes training conditions, program duration and training protocol, and (5) variables monitored. Data extraction was carried out by MZ, DB, TS and NA, while TK, BB and ZM checked the extracted data for accuracy and completeness, as well as ZM resolved disagreements if there was no consensus. Finally, selected studies were systematically reviewed by all authors, and the final check was made by TK and MZ.

\section{RESULTS}

Study selection. A search of electronic databases identified a total of 844 relevant studies. After removing the duplicates, 483 studies remained. Based on the review of the title and abstract, 242 studies were excluded, 121 studies were rejected due to different training in hypoxia conditions, 7 studies based on an inadequate number of participants and 97 dues to inadequate output data. According to clearly defined inclusion criteria, 14 studies met the inclusion criteria included in the systematic review and further analysis. The characteristics of the selected studies are shown in Table I, monitored variables in Table II, and the results are in Tables III and IV.

Study characteristics. All the studies included in this systematic review were published in English between February 2013 and March/April 2020. Most of studies were a parallel single-blind (i.e. Galvin et al.; Faiss et al., 2013; Gatterer et al., 2014; Goods et al., 2015; Kasai et al., 2015) or double-blind (i.e. Brocherie et al., 2015a, b; Faiss et al., 2015) design. The total sample size was 347 participants, of which 40 were females and 307 were males. 11 studies included only males (Galvin et al., Brocherie et al., 2015a; Faiss et al., 2013; Puype et al., 2013; Gatterer et al.; Brocherie et al., 2015b; Goods et al., 2015; Hamlin et al., 2017; Fornasier-Santos et al., 2018; Beard et al., 2019; Camacho-Cardenosa et al., 2020), one study included female participants (Kasai et al.), and two studies included participants of both sexes (Faiss et al., 2015; Brechbuhl et al., 2018). The age of the participants ranged from $15.3 \pm$ 0.5 to $35 \pm 7$ years. Four studies investigated the effects of repeated sprint training in hypoxia (RSH) in rugby players (Girard et al., 2013; Hamlin et al.; Fornasier-Santos et al.; Beard et al.), three in football players (Brocherie et al., 2015a; Gatterer et al.; Goods et al., 2015), two in cyclists (Faiss et al., 2015; Kasai et al.), and the rest of studies in hockey (Brocherie et al., 2015b), biathlon (Faiss et al., 2015) tennis (Brechbuhl et al.) and team sports (CamachoCardenosa et al.), while one study only mentioned term `athletes` (Puype et al.). The duration of the training program was varied from 12 days (Brechbuhl et al.) to 6 weeks (Puype et al.), and from 5 (Beard et al.) to 18 trainings (Puype et al.). Training conditions ranged for the $\mathrm{RSH}$ group from $13 \%$ to $14.8 \%$ the fraction of inhaled air $\left(\mathrm{FiO}_{2}\right)$, where they were most often trained at $14.5 \% \mathrm{FiO}_{2}$, and for the repeated sprint training in normoxia (RSN) group from $20.9 \%$ to $21 \% \mathrm{FiO}_{2}$ (in nine studies $20.9 \% \mathrm{FiO}_{2}$ ). For more details, see Table I.

The training protocol contained from one (Galvin et al.) to four (Brocherie et al., 2015b; Brechbuhl et al.) series of sprints and from five (Faiss et al., 2013; Gatterer et al.; Brocherie et al., 2015b; Faiss et al., 2015; Hamlin et al.; Brechbuhl et al.) up to ten (Galvin et al.) repetitions. Running time ranged from five seconds (Brocherie et al., 2015a, b) to ten seconds (Faiss et al., 2015; Gatterer et al.; Beard et al.; Camacho-Cardenosa et al.) or a sprint distance of 10 (Brocherie et al., 2015a) to 40 meters (Fornasier-Santos et al.). Also, the training protocol referred to pedaling on a bicycle ergometer, from one (Puype et al.) to four series 
$\underline{\text { Table I. Characteristics of the selected and analyzed studies. }}$

\begin{tabular}{|c|c|c|c|c|c|c|c|}
\hline \multirow[b]{2}{*}{ Author and year } & \multirow[t]{2}{*}{ Study design } & \multicolumn{2}{|r|}{ Sample of participants } & \multicolumn{3}{|c|}{ Experimental procedure } & \multirow[b]{2}{*}{ Variables } \\
\hline & & $\mathrm{N}$ & $\begin{array}{l}\text { Gender, age, groups, } \\
\text { sports }\end{array}$ & $\begin{array}{c}\text { Training } \\
\text { conditions }\end{array}$ & $\begin{array}{l}\text { Program } \\
\text { duration }\end{array}$ & $\begin{array}{l}\text { Training protocol (set } \mathrm{x} \text { repetition } \mathrm{x} \\
\text { duration / distance, rest between } \\
\text { repetitions, rest between sets) }\end{array}$ & \\
\hline Faiss et al. 2013 & $\begin{array}{l}\text { A parallel } \\
\text { single blind } \\
\text { design }\end{array}$ & 50 & $\begin{array}{c}\mathrm{M}=50 \\
35 \pm 7 \\
\mathrm{RSH} \mathrm{n}=20 \\
\mathrm{RSN} \mathrm{n}=20 \\
\text { CON } \mathrm{n}=20 \\
\text { cycling }\end{array}$ & $\begin{array}{c}\operatorname{RSH}(14.6 \% \\
\left.\mathrm{FiO}_{2}\right) \\
\sim 3000 \mathrm{~m} \\
\operatorname{RSN}(465 \mathrm{~m})\end{array}$ & $\begin{array}{c}4 \text { weeks } \\
8 \\
\text { workouts }\end{array}$ & $\begin{array}{l}3 \times 5 \times 10 \mathrm{~s} \text { (sprint), } 5 \mathrm{~min} \text { at } 120 \mathrm{~W} \text { on } \\
\text { the bike, at the end } \\
10 \mathrm{~min} \text { at } 120 \mathrm{~W} \text { on the bike }\end{array}$ & $\begin{array}{c}\text { RSAmax, RSAnum } \\
\text { (bicycle ergometer, } \\
10 \text { s pedaling, } 20 \mathrm{~s} \\
\text { active rest), } \\
\text { Wmean30s }\end{array}$ \\
\hline Galvin et al. 2013 & $\begin{array}{l}\text { A parallel } \\
\text { single-blind } \\
\text { design }\end{array}$ & 30 & $\begin{array}{c}M=30 \\
18.4 \pm 1.5 \\
\text { rugby } \\
M=29\end{array}$ & $\begin{array}{l}\mathrm{RSH}(13 \% \\
\left.\mathrm{FiO}_{2}\right) \\
\mathrm{RSN}^{2}(21 \% \\
\left.\mathrm{FiO}_{2}\right)\end{array}$ & $\begin{array}{l}4 \text { weeks, } \\
12 \\
\text { workouts }\end{array}$ & $1 \times 10 \times 6 \mathrm{~s}$ (sprint), $30 \mathrm{~s}$ & $\begin{array}{c}\text { RSAt, RSAd } \\
(10 x 20 \mathrm{~m}, 30 \mathrm{~s}) \\
\mathrm{VO}_{2 \max }, \mathrm{YYIR} 1\end{array}$ \\
\hline Puype et al. 2013 & $\begin{array}{l}\text { A parallel } \\
\text { single-blind } \\
\text { design }\end{array}$ & 29 & $\begin{array}{c}18-30 \\
\operatorname{RSH} n=10 \\
\operatorname{RSN} n=9 \\
\operatorname{CON} n=10\end{array}$ & $\begin{array}{c}\mathrm{RSH}(14.4 \% \\
\left.\mathrm{FiO}_{2}\right) \\
\operatorname{RSN}(20.9 \% \\
\left.\mathrm{FiO}_{2}\right)\end{array}$ & $\begin{array}{l}6 \text { weeks } \\
18 \text { training } \\
\text { sessions }\end{array}$ & $\begin{array}{l}\text { 1 x4-9 (+1 sprint per week }) \\
\times 30 \text { with pedaling } \\
\text { on a bicycle ergometer } \\
4 \text { min and } 30 \text { s pause }\end{array}$ & $\mathrm{VO}_{2 \max }$ \\
\hline $\begin{array}{l}\text { Gatterer et al. } \\
2014\end{array}$ & $\begin{array}{l}\text { A parallel } \\
\text { single-blind } \\
\text { design }\end{array}$ & 10 & $\begin{array}{c}M=10 \\
15.3 \pm 0.5 \\
\operatorname{RSH}(\mathrm{n}=5) \\
\operatorname{RSN}(\mathrm{n}=5) \\
\text { football } \\
\mathrm{M}=32\end{array}$ & $\begin{array}{c}\mathrm{RSH}(14.8 \% \\
\left.\mathrm{FiO}_{2}\right) \\
\sim 3300 \mathrm{~m} \\
\mathrm{RSN}(20.9 \% \\
\left.\mathrm{FiO}_{2}\right) \\
\sim 200 \mathrm{~m}\end{array}$ & $\begin{array}{l}5 \text { weeks, } \\
10 \\
\text { workouts } \\
\text { (at least } 7 \\
\text { trainings) }\end{array}$ & $3 \times 5 \times 10 \mathrm{~s}$ (sprint), $20 \mathrm{~s}, 5 \mathrm{~min}$ & $\begin{array}{l}\text { RSAt (6x40 m, } 20 \mathrm{~s} \\
\text { passive rest), } \\
\text { YYIR2 (RSH n }=4 \text { ) }\end{array}$ \\
\hline $\begin{array}{l}\text { Brocherie, Millet } \\
\text { ot al. } 2015\end{array}$ & $\begin{array}{l}\text { A parallel } \\
\text { double-blind } \\
\text { design }\end{array}$ & 32 & $\begin{array}{c}25.3 \pm 4.6 \\
\operatorname{RSH} \mathrm{n}=11 \\
\mathrm{RSN} \mathrm{n}=12 \\
\text { CON } \mathrm{n}=9 \\
\text { field hockey }\end{array}$ & $\begin{array}{c}\mathrm{RSH}(14.5 \% \\
\left.\mathrm{FiO}_{2}\right) \\
\sim 3000 \mathrm{~m} \\
\mathrm{RSN}(21.0 \% \\
\left.\mathrm{FiO}_{2}\right)\end{array}$ & $\begin{array}{c}2 \text { weeks } \\
6 \\
\text { workouts }\end{array}$ & $\begin{array}{l}4 \times 5 \times 5 \mathrm{~s} \text { (sprint), } 25 \mathrm{~s}, \\
5 \text { min passive rest }\end{array}$ & $\begin{array}{c}\text { RSAt } \\
(8 \times 20 \mathrm{~m}, 20 \mathrm{~s}) \\
\text { YYIR2 }\end{array}$ \\
\hline $\begin{array}{l}\text { Brocherie } \text { et al. } \\
2015\end{array}$ & $\begin{array}{c}\text { A parallel } \\
\text { double-blind } \\
\text { design }\end{array}$ & 16 & $\begin{array}{c}\mathrm{M}=16 \\
\mathrm{RSH}(\mathrm{n}=8) 17.1 \pm 0.3 \\
\mathrm{RSN}(\mathrm{n}=8) 17.1 \pm 0.2 \\
\text { football }\end{array}$ & $\begin{array}{c}\mathrm{RSH}(14.3 \% \\
\left.\mathrm{FiO}_{2}\right) \\
\sim 2900 \mathrm{~m} \\
\mathrm{RSN}(21.0 \% \\
\left.\mathrm{FiO}_{2}\right)\end{array}$ & $\begin{array}{l}5 \text { weeks, } \\
10 \\
\text { workouts }\end{array}$ & $\begin{array}{l}\text { 4-6x3-4x5 or } 10 \mathrm{~m} \text { (sprint, } \\
\text { explosive strenght), } 45 \mathrm{~s}, 3 \text { min, } \\
\text { HI 2-3x5-6x15 } \\
\text { (treadmill), } 15 \mathrm{~s} \text { passive rest, } 5-10 \\
\text { min light running }\end{array}$ & $\begin{array}{l}\text { RSAt }(10 \times 30 \mathrm{~m}, 30 \\
\quad \text { s passive rest) }\end{array}$ \\
\hline Faiss et al. 2015 & $\begin{array}{l}\text { A parallel } \\
\text { double-blind } \\
\text { design }\end{array}$ & 17 & $\begin{array}{c}\mathrm{M}=11, \mathrm{~F}=6 \\
\mathrm{M}=29.6 \pm 8.8 \\
\mathrm{~F}=23.4 \pm 5 \\
\mathrm{RSH} \mathrm{n}=9 \\
\mathrm{RSN} \mathrm{n}=8 \\
\text { biathlon }\end{array}$ & $\begin{array}{l}\mathrm{RSH}(13.8 \% \\
\mathrm{FiO})_{2} \\
\sim 3000 \mathrm{~m} \\
\operatorname{RSN}(20.9 \% \\
\left.\mathrm{FiO}_{2}\right) \\
\sim 200 \mathrm{~m}\end{array}$ & $\begin{array}{c}2 \text { weeks } \\
6 \\
\text { workouts }\end{array}$ & $\begin{array}{l}4 \times 5 \times 10 \mathrm{~s} \text { (pedaling), } 20 \mathrm{~s}, \\
4 \mathrm{~min} \text { and } 50 \mathrm{~s} \text { active rest }\end{array}$ & $\begin{array}{l}\text { RSAmax, RSAnum, } \\
\text { RSAmean (10 s } \\
\text { sprint, } 20 \text { s break- } \\
\text { modified biathlon } \\
\text { ergometer })\end{array}$ \\
\hline Goods et al. 2015 & $\begin{array}{l}\text { A parallel } \\
\text { single-blind } \\
\text { design }\end{array}$ & 30 & $\begin{array}{c}M=30 \\
20.3 \\
\operatorname{RSH} n=10 \\
\operatorname{RSN} n=10 \\
\operatorname{CON} n=10\end{array}$ & $\begin{array}{c}\mathrm{RSH}(14.5 \% \\
\left.\mathrm{FiO}_{2}\right) \\
\sim 3000 \mathrm{~m} \\
\mathrm{RSN}(20.9 \% \\
\left.\mathrm{FiO}_{2}\right) \\
\sim 200 \mathrm{~m}\end{array}$ & $\begin{array}{c}5 \text { weeks } \\
15 \\
\text { workouts }\end{array}$ & $\begin{array}{l}3 \times 7 \times 5 \mathrm{~s} \text { (pedaling) ( }+1 \text { sprint per } \\
\text { week), } 15-35 \mathrm{~s}, 3 \text { min active rest }\end{array}$ & $\begin{array}{c}\text { RSAt } \\
\text { (3x6x20 m, 25 s, } 3 \\
\text { min) } \\
\text { RSAmax, RSAmean } \\
\text { (pedaling 3x6x4 s, } \\
25 \mathrm{~s}, 3 \text { min) }\end{array}$ \\
\hline Kasai et al. 2015 & $\begin{array}{l}\text { A parallel } \\
\text { single-blind } \\
\text { design }\end{array}$ & 32 & $\begin{array}{c}\text { football } \\
\quad \mathrm{F}=32 \\
\operatorname{RSH}(\mathrm{n}=16) 20.0 \pm 0.2 \\
\operatorname{RSN}(\mathrm{n}=16) 20.0 \pm 0.1 \\
\text { cycling }\end{array}$ & $\begin{array}{c}\mathrm{RSH}(14.5 \% \\
\left.\mathrm{FiO}_{2}\right) \\
\sim 3000 \mathrm{~m} \\
\sim \operatorname{RSN}(20.9 \% \\
\left.\mathrm{FiO}_{2}\right)\end{array}$ & $\begin{array}{l}4 \text { weeks, } \\
8 \\
\text { workouts }\end{array}$ & $2 \times 10 \times 7 \mathrm{~s}$ (pedaling), $30 \mathrm{~s}$ rest & $\begin{array}{c}\text { RSAmax, RSAmean } \\
\text { pedaling }(10 x 7 \mathrm{~s}), \\
\operatorname{VO}_{2 \max }\end{array}$ \\
\hline Hamlin et al. 2017 & $\begin{array}{l}\text { A parallel } \\
\text { single-blind } \\
\text { design }\end{array}$ & 19 & $\begin{array}{c}\mathrm{M}=19 \\
\mathrm{RSH}(\mathrm{n}=8) \\
20.3 \pm 2.1 \\
\mathrm{RSN}(\mathrm{n}=10) \\
22.0 \pm 4.1 \\
\text { rugby }\end{array}$ & $\begin{array}{l}\mathrm{RSH}(14.5 \% \\
\mathrm{FiO} \mathrm{O}_{2} \\
\sim 300 \mathrm{~m} \\
\mathrm{RSN}(20.9 \% \\
\left.\mathrm{FiO} \mathrm{O}_{2}\right) \\
\sim 200 \mathrm{~m}\end{array}$ & $\begin{array}{l}4 \text { weeks, } \\
8 \\
\text { workouts }\end{array}$ & $\begin{array}{c}4 \times 5 \times 5 \mathrm{~s} \text { (pedaling), } 25 \mathrm{~s} \text { active rest } \\
\text { at } \sim 20-50 \mathrm{~W}, 5 \text { min active rest } \\
\text { slow pedaling at } \sim 20-50 \mathrm{~W} \text { on a } \\
\text { bicycle ergometer }\end{array}$ & $\begin{array}{c}\text { RSAt } \\
(8 \times 20 \mathrm{~m}, 20 \mathrm{~s}) \\
\text { YYIR1 }\end{array}$ \\
\hline $\begin{array}{l}\text { Brechbuhl et al. } \\
2018\end{array}$ & $\begin{array}{l}\text { A parallel } \\
\text { single-blind } \\
\text { design }\end{array}$ & 18 & $\begin{array}{c}\mathrm{M}=16, \mathrm{~F}=2 \\
\operatorname{RSH}(\mathrm{n}=9, \mathrm{M}=8, \mathrm{~F}=1) \\
24.8 \pm 5.1 \\
\operatorname{RSN}(\mathrm{n}=9, \mathrm{M}=8, \mathrm{~F}=1) \\
22.8 \pm 4.1 \\
\text { tennis } \\
\mathrm{M}=21\end{array}$ & $\begin{array}{c}\mathrm{RSH}(14.5 \% \\
\left.\mathrm{FiO}_{2}\right) \\
\sim 300 \mathrm{~m} \\
\mathrm{RSN}(20.9 \% \\
\left.\mathrm{FiO} \mathrm{O}_{2}\right) \\
\sim 200 \mathrm{~m}\end{array}$ & $\begin{array}{l}12 \text { days, } \\
5 \\
\text { workouts }\end{array}$ & $4 \times 5 \times 6 \mathrm{~s}$ (sprint), $24 \mathrm{~s}, 4.5 \mathrm{~min}$ & $\begin{array}{l}\text { RSAt (10x20 m, } 20 \\
\text { s), } \mathrm{VO}_{2 \max }\end{array}$ \\
\hline $\begin{array}{l}\text { Fornasier-Santos } \\
\text { et al. } 2018\end{array}$ & $\begin{array}{l}\text { A parallel } \\
\text { double-blind } \\
\text { design }\end{array}$ & 21 & $\begin{array}{c}18.3 \pm 1.3 \\
\operatorname{RSH}(\mathrm{n}=11) \\
\operatorname{RSN}(\mathrm{n}=10) \\
\text { rugby }\end{array}$ & $\sim 2000 \mathrm{~m}$ & $\begin{array}{l}4 \text { weeks, } \\
8 \\
\text { workouts }\end{array}$ & $\begin{array}{l}2 \times 8 \text { ( }+2 \text { sprints per week) } \times 40 \mathrm{~m}, 30 \\
\mathrm{~s}, 3 \text { min semi-active rest (walking) }\end{array}$ & $\begin{array}{l}\text { RSAnum ( } 40 \mathrm{~m}, 30 \\
\mathrm{~s})\end{array}$ \\
\hline Beard et al. 2019 & $\begin{array}{l}\text { A parallel } \\
\text { double-blind } \\
\text { design }\end{array}$ & 19 & $\begin{array}{c}\mathrm{M}=19 \\
\mathrm{RSH}(\mathrm{n}=10) \\
25.6 \pm 3.7 \\
\mathrm{RSN}(\mathrm{n}=9) \\
23.0 \pm 1.9 \\
\text { rugby }\end{array}$ & $\begin{array}{c}\mathrm{RSH}(13.8 \% \\
\left.\mathrm{FiO}_{2}\right) \\
\mathrm{RSN}_{2}(20.9 \% \\
\left.\mathrm{FiO}_{2}\right) \\
\sim 200 \mathrm{~m}\end{array}$ & $\begin{array}{c}2 \text { weeks, } \\
5 \\
\text { workouts }\end{array}$ & $3 \times 8 \times 10 \mathrm{~s}, 20 \mathrm{~s}$ passive rest, $2 \mathrm{~min}$ & $\begin{array}{l}\text { RSAmean, RSAmax } \\
\text { (pedaling } 6 \times 10 \mathrm{~s}, 20 \\
\mathrm{~s} \text { ) }\end{array}$ \\
\hline $\begin{array}{l}\text { Camacho- } \\
\text { Cardenosa et al. } \\
2020\end{array}$ & $\begin{array}{l}\text { A parallel } \\
\text { double-blind } \\
\text { design }\end{array}$ & 24 & $\begin{array}{c}\mathrm{M}=24 \\
\mathrm{RSH}(\mathrm{n}=8) 23.1 \pm 2.5 \\
\mathrm{RSN}(\mathrm{n}=8) 22.5 \pm 2.9 \\
\mathrm{CON}(\mathrm{n}=8) 22.7 \pm 3.2 \\
\quad \text { team sports }\end{array}$ & $\begin{array}{c}\mathrm{RSH}(14.6 \% \\
\left.\mathrm{FiO}_{2}\right) \\
\sim 3400 \mathrm{~m} \\
\sim 34 \mathrm{R}^{2}(20.9 \% \\
\mathrm{FiO}, \\
\sim 200 \mathrm{~m}\end{array}$ & $\begin{array}{l}4 \text { weeks, } \\
8 \\
\text { workouts }\end{array}$ & $\begin{array}{c}2 \times 5 \times 10 \mathrm{~s} \text { (sprint), } 20 \mathrm{~s}, 10 \mathrm{~min} \text { at } \\
120 \mathrm{~W}, \\
\text { bicycle ergometer }\end{array}$ & $\begin{array}{c}\text { RSAnum (for } 10 \mathrm{~s} \\
\text { maximum pedaling, } \\
20 \mathrm{~s} \text { active rest at } \\
120 \mathrm{~W} \text { ), Wmax } 30 \mathrm{~s} \\
\text { (bicycle ergometer), } \\
\text { YYIR } 1, \mathrm{VO}_{\operatorname{mmax}}\end{array}$ \\
\hline
\end{tabular}

$\mathrm{N}$ number of participants, $\mathrm{n}$ subsample, $\mathrm{M}$ male, $\mathrm{F}$ female, $\mathrm{FiO}_{2}$ oxygen concentration in inhaled volume, RSH repeated-sprint hypoxia, RSN repeated-sprint normoxia, CON control group, HI high-intensity training, RSA repeated-sprint abilities, RSAt repeated -sprint abilities, total time, RSAnum repeated-sprint abilities, number of sprints, RSAd repeated-sprint abilities, distance traveled, RSAmax repeated-sprint abilities, maximum strength, RSAmean repeated-sprint abilities, mean power, $\mathrm{VO}_{2 \max }$ maximum oxygen consumption, Wmax30s Wingate test maximum power for 30s, Wmean30s Wingate test mean power for 30s, YYIR1, YYIR2 interval recovery test, Post transit measurement.
(Faiss et al., 2015; Hamlin et al.), from five (Faiss et al., 2015; Hamlin et al.) to ten repetitions (Kasai et al.), in the study by Puype et al. there was a progressive increase in the number of repetitions during six weeks of training, from four at the beginning to nine repetitions at the end of the protocol) and a pedaling time of five (Goods et al., 2015; Hamlin et al.) to ten seconds (Faiss et al., 2015). The exception was the study of Brocherie et al. (2015a), where the sprint series were defined from four to six, the number of repetitions from three to four and the running time of five seconds or the distance covered by ten meters.

All studies, which were included in the systematic review, aimed to examine the statistical significance $(\mathrm{p}<0.05$, $p<0.01$ and $p<0.001)$ of the effect of RSH on the physical components among athletes, using a total of ten monitored variables related to repeated sprint ability (RSA): time (RSAt), number of sprints to exhausted (RSAnum), distance travelled (RSAd), maximum or average power produced (RSAmax, RSAmean) during RSA test, maximum and average power produced during Wingate test (Wmax30s, Wmean30s), maximum consumption oxygen (VO2max), as well as the results obtained using Yo-Yo tests (YYIR1, YYIR2). The number of monitored variables ranged from one (Brocherie et al., 2015a; Puype et al.; FornasierSantos et al.) to four (Galvin et al.; Camacho-Cardenosa et al.). The total number of monitored variables and their representation in studies are shown in Table II. 
Table II. Monitored variables and representation in studies.

\begin{tabular}{|c|c|c|c|c|c|c|c|c|c|c|c|}
\hline \multirow{2}{*}{ Author and year } & \multicolumn{11}{|c|}{ Variables } \\
\hline & RSAt & RSAd & RSAnum & RSAmax & RSAmean & $\mathrm{Wmax} 30 \mathrm{~s}$ & Wmean30s & $\mathrm{VO}_{2 \max }$ & YYIR1 & YYIR2 & \\
\hline Faiss et al. 2013 & & & + & + & & & + & & & & 3 \\
\hline Galvin et al. 2013 & + & + & & & & & & + & + & & 4 \\
\hline Puype et al. 2013 & & & & & & & & + & & & 1 \\
\hline Gatterer et al. 2014 & + & & & & & & & & & + & 2 \\
\hline Brocherie et al. 2015 & + & & & & & & & & & + & 2 \\
\hline Brocherie et al. 2015 & + & & & & & & & & & & 1 \\
\hline Faiss et al. 2015 & & & + & + & + & & & & & & 3 \\
\hline Goods et al. 2015 & + & & & + & + & & & & & & 3 \\
\hline Kasai et al. 2015 & & & & + & + & & & + & & & 3 \\
\hline Hamlin et al. 2017 & + & & & & & & & & + & & 2 \\
\hline Brechbuhl et al. 2018 & + & & & & & & & + & & & 2 \\
\hline Fornasier-Santos et al. 2018 & & & + & & & & & & & & 1 \\
\hline Beard et al. 2019 & & & & + & + & & & & & & 2 \\
\hline Camacho-Cardenosa et al. 2020 & & & + & & & + & & + & + & & 4 \\
\hline & 7 & 1 & 4 & 5 & 4 & 1 & 1 & 5 & 3 & 2 & \\
\hline
\end{tabular}

RSAt repeated-sprint abilities, total time, RSAd repeated-sprint abilities, distance traveled, RSAnum repeated-sprint abilities, number of sprints, RSAmax repeated-sprint abilities, maximum power, RSAmean repeated-sprint abilities, average power, Wmax30s Wingate test maximum power for 30s, Wmean30s Wingate test mean power value for 30s, VO2max maximum oxygen consumption, YYIR1, YYIR2 interval recovery test.

Study results. In a detailed analysis of the procedures and results of RSH studies was found that the studies were mainly conducted with participants who were active athletes (rugby, football, cycling, field hockey, tennis, etc.). In seven studies, one of the aims was to examine the impact of RSH training at the time of pedaling during the test to assess the ability to perform a repeated sprint (RSAt). The positive impact of RSH was evident in all participants in six studies (Galvin et al.; Gatterer et al.; Brocherie et al., 2015a, b; Goods et al., 2015; Hamlin et al.; Brechbuhl et al.). However,

Table III. Results of analyzed studies for RSA.

\begin{tabular}{|c|c|c|c|c|c|}
\hline Author and year & RSAt & RSAd & RSAnum & RSAmax & RSAmean \\
\hline \multirow{3}{*}{ Faiss et al., 2013} & & & RSH $13.06 \pm 6.2 \uparrow * *$ & RSH $6 \pm 7 \% \uparrow * *$ & \\
\hline & & & $\operatorname{RSN} 8.96 \pm 3.5 \downarrow$ & $\operatorname{RSN} 7 \pm 8 \% \uparrow * *$ & \\
\hline & & & $\mathrm{CON} 10.36 \pm 6.2 \downarrow$ & $\mathrm{CON} 2 \pm 5 \% \uparrow$ & \\
\hline \multirow{2}{*}{ Galvin et al., 2013} & $\mathrm{RSH} 2.7 \pm 1.6 \% \uparrow *$ & $\mathrm{RSH}+15.1 \mathrm{~m}$ & & & \\
\hline & RSN $3.7 \pm 2.1 \% \uparrow *$ & $\mathrm{RSN}+6.5 \mathrm{~m}$ & & & \\
\hline \multirow{2}{*}{ Gatterer et al., 2013} & $\mathrm{RSH} 7.4 \pm 0.3 ; 7.3 \pm 0.2 \uparrow$ & & & & \\
\hline & $\operatorname{RSN} 7.6 \pm 0.3 ; 7.6 \pm 0.2 \uparrow$ & & & & \\
\hline \multirow[b]{2}{*}{ Brocherie etal., 2015} & RSH3.6\% $\% * * * \operatorname{RSN} 1.9 \% \uparrow * *$ & & & & \\
\hline & CON $0.7 \% \uparrow$ & & & & \\
\hline \multirow{2}{*}{ B rocherie et al., 2015} & RSH-3.2 $\pm 1.7 \% \uparrow * *$ & & & & \\
\hline & RSN-1.9 $\pm 2.6 \% \uparrow *$ & & & & \\
\hline \multirow{2}{*}{ Faiss etal., 2015} & & & RSH $17.1 \pm 6.8 \uparrow * *$ & RSH $29 \% \pm 13 \%$ 个** & $\mathrm{RSH} 11 \pm 9 \% \uparrow * *$ \\
\hline & & & RSN $11.7 \pm 4.3 \uparrow$ & $\operatorname{RSN} 26 \% \pm 18 \% \uparrow * *$ & $\operatorname{RSN} 15 \pm 7 \% \uparrow * *$ \\
\hline \multirow{3}{*}{ Goods et al., 2015} & RSH $2.3 \% \uparrow \operatorname{RSN~} 1.8 \% \uparrow$ & & & RSH $4.7 \% \uparrow * * *$ & RSH $10.3 \% \uparrow * * *$ \\
\hline & $\mathrm{CON} 1.1 \% \uparrow$ & & & RSN $8.6 \% \uparrow * *$ & RSN $13.6 \% \uparrow * * *$ \\
\hline & & & & CON $1.1 \% \downarrow$ & $\mathrm{CON} 1.4 \% \uparrow$ \\
\hline \multirow{2}{*}{ Kasai et al., 2015} & & & & RSH $5.0 \pm 0.7 \% \uparrow *$ & RSH $9.7 \pm 0.9 \% \uparrow *$ \\
\hline & & & & $\mathrm{RSN} 1.5 \pm 0.9 \% \uparrow *$ & $\operatorname{RSN} 6.0 \pm 0.8 \% \uparrow *$ \\
\hline Hamlin et al., 2017 & $\begin{array}{c}\text { Post } 3: \\
\text { RSH } 1.0 \pm 4.5 \% \text { RS } * \text { RSN } 1.7 \pm 4.1 \% \uparrow * \\
\text { Post } 4: \\
\text { RSH } 1.3 \pm 4.5 \% \text { RS } * \text { RSN } 0.4 \pm 4.4 \% \uparrow *\end{array}$ & & & & \\
\hline B rechbuhl et al., 2018 & RSH $1.1 \% \uparrow \operatorname{RSN} 0.8 \% \uparrow$ & & & & \\
\hline Fornasier-Santos et al. & & & RSH $64 \% \uparrow * *$ & & \\
\hline 2018 & & & RSN has no changes & & \\
\hline \multirow{2}{*}{ B eard et al., 2019} & & & & $\begin{array}{c}\text { RSH } 12.84 \pm 0.83 \text { Vs. } \\
13.63 \pm 1.03 \uparrow *\end{array}$ & $\begin{array}{c}\text { RSH } 11.15 \pm 0.58 \text { vs. } \\
11.86 \pm 0.63\end{array}$ \\
\hline & & & & RSN has no changes & RSN has no changes \\
\hline $\begin{array}{l}\text { Camacho-Cardenosa et } \\
\text { al., } 2020\end{array}$ & & & RSH $20.36 \% \uparrow$ & & \\
\hline
\end{tabular}

RSH repeated-sprint hypoxia, RSN repeated-sprint normoxia, CON control group, RSA repeated-sprint abilities, RSAt repeated-sprint abilities, total time, RSAnum repeated-sprint abilities, number of sprints, RSAd repeated-sprint abilities, distance traveled, RSAmax repeated -sprint ability, maximum strength, RSAmean repeated-sprint ability, mean strength, $\neq * \mathrm{p} ? 0.05, \neq * * \mathrm{p} ? 0.01, \neq * * * \mathrm{p}<0.001$. 
statistically significant progress among all participants was occurred in three studies (Galvin et al.; Brocherie et al., 2015a, b;), while one study indicated statistically significant progress in only one group (Hamlin et al.). The distance covered during the repeated sprint test (RSAd) was examined only in one study included in this systematic review in which statistically significant progress was found (experimental group: + $15.1 \mathrm{~m}$, control group: $+6.5 \mathrm{~m} ; \mathrm{p}<0.05)$ in both groups of participants (Galvin et al.). A statistically significant difference in the experimental group before and after performing $\mathrm{RSH}$, which is related to the number of sprints to exhaustion during the test to assess the ability to perform repeated sprints (RSAnum), occurred in four of the five studies. The values obtained after the program in the experimental group were increased from $13.06 \pm 6.2$, $\mathrm{p}<$ 0.05 (Faiss et al., 2013) to $17.1 \pm 6.8, \mathrm{p}<0.01$ (Faiss et al., 2015), while the percentage progress was $64 \%$, p <0.01 (Fornasier-Santos et al.). Performing a test to assess the RSA, where during testing the emphasis was placed on the results related to the produced average or maximum strength (RSAmean, RSAmax) was a task in five studies, in which statistically significant progress was found (Faiss et al., 2013; 2015; Goods et al., 2015; Kasai et al.; Beard et al.). For more details, see Table III.
In order to assess the impact of RSH training method on the produced maximum or average strength, the authors of two studies included in this systematic review used the Wingate test for 30s as a testing protocol (Faiss et al., 2013; Camacho-Cardenosa et al.) where progress was found in both studies, but without statistical significance ( $p>0.05)$. For more details, see Table IV.

The effect of RSH training method on the improvement of maximum oxygen consumption, for which great heterogeneity was found in the conclusions, was examined in five studies. Progress without statistical significance was found in three studies (Galvin et al.; Brechbuhl et al.; Camacho-Cardenosa et al.). Puype et al., who tested VO2max in their study using the maximum incremental test in hypoxia and normoxia, indicated a statistically significant progress of the experimental group of $6 \%$ to $8 \%$, while one study did not find any changes after conducting RSH (Kasai et al.). For more details, see Table IV.

The values obtained during the Yo-Yo tests (YYIR1, YYIR2) were tested in five studies and all indicated the improvement of the distance covered during the test, and

Table IV. Results of analyzed studies for Wingate, $\mathrm{VO}_{2 \max }$ and YYIR.

\begin{tabular}{|c|c|c|c|c|c|}
\hline Author and year & $\mathrm{Wmax} 30 \mathrm{~s}$ & Wmean30s & $\mathrm{VO}_{2 \max }$ & YYIR1 & YYIR2 \\
\hline Faiss et al., 2013 & & $\begin{array}{l}\text { RSH 718 } \pm 94 \uparrow ; \\
\text { RSN } 723 \pm 86 \uparrow \\
\text { CON } 689 \pm 105 \uparrow\end{array}$ & & & \\
\hline Galvin et al., 2013 & & & $\begin{array}{c}\text { RSH } 6.9 \pm 9 \% \uparrow \\
\text { RSN }-0.3 \pm 8.8 \% \downarrow\end{array}$ & $\begin{array}{c}\text { RSH } 33 \pm 12 \% \uparrow * * \\
\text { RSN } 14 \pm 10 \% \uparrow\end{array}$ & \\
\hline Puype et al., 2013 & & & $\begin{array}{c}\text { RSH, RSN } 6 \%-8 \% \uparrow * \\
\text { CON has no changes }\end{array}$ & & \\
\hline Gatterer et al., 2014 & & & & & $\begin{array}{c}\text { RSH } 1430 \pm 458 ; 1710 \pm \\
183 \\
\text { RSN } 1832 \pm 310 ; 2216 \pm\end{array}$ \\
\hline Brocherie et al., 2015 & & & & & $\begin{array}{c}\operatorname{RS} 19.21 \% \\
\operatorname{RSN} 22 \% \uparrow * * * \\
\operatorname{CON} 4 \% \uparrow\end{array}$ \\
\hline Kasai et al., 2015 & & & RSH, RSN has no changes & & \\
\hline Hamlin et al., 2017 & & & & $\begin{array}{c}\text { RSH Post 3-4-5 } \\
26 \pm 31 \% \uparrow * \\
37 \pm 31 \% \uparrow * \\
33 \pm 29 \% \uparrow * \\
\text { RSN Post } 1-2-4-5 \\
20 \pm 26 \% \uparrow * \\
26 \pm 26 \% \uparrow * \\
26 \pm 28 \% \uparrow *\end{array}$ & \\
\hline Brechbuhl et al., 2018 & & & RSH $0.8 \% \uparrow$ RSN $-1.7 \% \downarrow$ & & \\
\hline Camacho-Cardenosa et al., 2020 & RSH $14.96 \% \uparrow$ & & RSH $5.89 \% \uparrow$ & RSH $15.8 \% \uparrow$ & \\
\hline
\end{tabular}


after the experimental procedure. Galvin et al., indicated a statistically significant progress of the experimental group of $33 \pm 12 \%$. Gatterer et al., found that the participants of the experimental and control groups statistically significantly improved their values before and after the implementation of the program (experimental group; before: $1430 \pm 458$; after: $1710 \pm 183$; control group; before: $1832 \pm 310$; after $2216 \pm$ $395)$. Statistically significant progress of the experimental and control groups $(15-33 \%)$ was found in three studies (Brocherie et al., 2015b; Hamlin et al.; Camacho-Cardenosa et al.). For more details, see Table III.

\section{DISCUSSION}

The purpose of this systematic review was to analyze the selected studies with a current topic related to repeated sprint training in hypoxia (RSH) and its effect on the repeated sprint ability (RSA), $\mathrm{VO}_{2 \max }$, as well as on performance in Wingate, and Yo-Yo (YYIR1/YYIR2) tests. The results of the selected and systematically reviewed studies showed that RSH was an effective method in improving the ability to perform different modalities of RSA, Wingate, $\mathrm{VO}_{2 \max }$, and Yo-Yo (YYIR1/YYIR2) tests. However, major improvements were observed mainly inRSA tests, and less in aerobic tests (i.e. Wingate and Yo-Yo). Furthermore, it is important to notice that RSH groups generally achieved better results in physical performance compared to repeated sprint training in normoxia (RSN) and control groups. These results were line with the previous systematic reviews and meta-analyses which suggested that RSH was more effective training method for improving sport-specific performance compared to RSN among athletes, and RSH induced greater gains in RSA (Brocherie et al., 2017; Millet et al.).

RSH has been considered effective in improving RSA, which has been recognized to be very similar to the requirements of team sports in real game conditions (Camacho-Cardenosa et al.). The most common modality of the RSA test was with the measurement of total time during maximum repetitive sprints (RSAt) (Galvin et al.; Gatterer et al.; Brocherie et al., 2015a, b; Goods et al., 2015; Hamlin et al.). Direct comparison of the results of the mean values of the RSAt test was difficult due to the fact that in each of these studies applied different testing protocols, ie variations in the number of series, repetitions, distance, as well as the duration and nature of rest between repetitions. However, observing the results of selected studies and changes recorded in percentages (Brocherie et al., 2015a, b; Goods et al., 2015; Hamlin et al.), it could be suggested that the progress of RSH groups was significantly higher compared to RSN (1.0 - 3.6\% vs. - $1.7-1.9 \%$, respectively). Physiological mechanisms such as improved phosphocreatine resynthesis rate and increased mitochondrial activity that occur in the body's adaptation to high-intensity training, such as repeated sprint training in hypoxia, may be responsible for achieving better RSH group results (Bishop \& Girard, 2013). Also, it is considered that better results in RSH are a consequence of muscle adaptation at the molecular level in the form of higher oxidative capacity and glycolytic muscle potential, as well as more efficient use of fast muscle fibers (Faiss et al., 2013). In other studies, there were observed shifts in both groups of participants, but no significant differences between them were observed (Galvin et al.; Brocherie et al., 2015a; Goods et al., 2015), while there were no significant differences between pre- and post-treatment testing in either group (Gatterer et al.; Brechbuhl et al.).

The second modality of the RSA test in terms of prevalence is performed on a bicycle ergometer, which gives the values of maximum output power (RSAmax) and mean power values (RSAmean). The results of the RSA test on a bicycle ergometer in most studies (Faiss et al., 2015; Goods et al., 2015; Kasai et al.) showed superior percentage values of RSAmax in RSH compared to RSN (4.7 - $29 \%$ vs. $1.5-26$ $\%$, respectively), while in the study Beard et al. significant progress was observed only in the RSH group $(6.2 \%)$. Maximum strength production during repeated sprints is associated with the ability to recruit more fast-twitch muscle fibers at very high pedaling frequencies, and fast-twitch muscle fibers are known to be crucial in strength production when increasing intensity (Gollnick et al., 1974). Consequently, the observed increase in maximum power could be probably attributed to improved muscle recruitment coordination in terms of recruiting more fast-fiber motor units as well as improved intermuscular coordination (Billaut $e t$ al., 2005).

In contrast to the previous findings, the RSN group made greater progress compared to RSH (7 - 8.6 vs. 4.7 - $6 \%$, respectively) in two studies (Faiss et al., 2013; Goods et al., 2015). This might be a consequence of the reduced achieved training intensity in hypoxia conditions compared to normoxia conditions (Goods et al., 2014). The RSA mean parameters related to the mean power values expressed in the test showed a larger shift of the RSN group compared to RSH (Faiss et al., 2013; 2015; Goods et al., 2015). In contrast, Kasai et al., found that the achieved RSA mean results favored RSH compared to RSN (9.7 vs. $6 \%$, respectively), while Beard et $a l$. observed a shift only in RSH $(6.4 \%)$.

The results achieved in the RSA test, which is realized in relation to the number of completed sections - sprints to exhaustion (RSAnum), indicated that significant improvements in this test were visible only in RSH groups 
(20 - 64\%) (Faiss et al., 2013; 2015; Fornasier-Santos et al.; Camacho-Cardenosa et al.). The higher number of repeated RSAnum sprints, in RSH, could be explained by improved fast muscle fiber behavior, although this requires optimization of anaerobic glycolysis that is impaired due to sprint repetition (Girard et al., 2011). In addition, compensatory vasodilation (dilation of blood vessels) occurs during exercise in hypoxia, which allows muscle tissue to receive more blood, which somewhat compensates for hypoxia and aims to maintain a constant supply of oxygen to the muscles (Casey \& Joyner, 2012). During repeated sprints, the breakdown of phosphocreatine is very high, and inorganic phosphate accumulates in the muscles (Bogdanis et al., 1996). Because increased phosphate levels can cause a decrease in the ability to produce strength in fast-twitch muscle fibers, especially during such strenuous exercise (Westerblad et al., 2002), increased blood flow can promote faster removal of waste metabolites and may delay fatigue during the RSA test.

Only Galvin et al. presented the results of the RSA test in which the total distance traveled (RSAd) was calculated, where the participants of both study groups achieved significantly better results compared to the initial measurement (RSH $3.4 \%$; RSN $1.5 \%$ ), but it should be noted that no significant intergroup differences were found. This improvement in performance might be due to less cerebral deoxygenation resulting from hypoxic training, as cerebral deoxygenation is thought to be associated with central fatigue (Goodall et al., 2012).

Wingate test results showed a more significant increase in mean power (Wmean $30 \mathrm{~s}$ ) in RSN (5\%) compared to RSH (2.7\%) (Faiss et al., 2013), but without significant differences between groups. Previous findings suggested that repeated sprint training in hypoxic conditions was not effective enough in endurance athletes (Lundby \& Robach, 2016). In the variable maximum expressed power (Wmax $30 \mathrm{~s}$ ), a significant increase $(15 \%)$ was recorded only in the RSH group (Camacho-Cardenosa et al.). Consequently, related to VO2max. Puype et al. observed that there was a statistically significant progress of the experimental groups RSH and RSN (6\% and $8 \%$, respectively), while no changes were observed after conducting RSH. In some studies, there were changes in $\mathrm{VO}_{2 \max }$, to a greater extent in $\mathrm{RSH}$, but without statistical significance (Galvin et al.; Brechbuhl et al.; CamachoCardenosa et al.).

Athletic performance in YYIR1 and YYIR2 tests correlated positively with sports-specific performance such as rugby, football, handball, basketball, etc. (Galvin et al.). Training with repeated sprints on the treadmill caused significant improvements after four weeks (12 trainings) stocks covered during the YYIR1 test code RSH groups (33\%) compared to RSN (14\%) (Galvin et al.). The proposed program was more effective compared to traditional repetitive sprint training that lasts longer (seven weeks) (Galvin et al.). In contrast to these improvements, hypoxic conditions did not lead to significant improvements in YYIR2 test scores compared to normoxic conditions (Gatterer et al.; Brocherie et al., 2015b; Hamlin et al.; Camacho-Cardenosa et al.). According to previous studies, muscle oxidative activity might be, to a lesser extent, susceptible to this type of training, and to some extent explains why there was no effect of hypoxia on YYIR results (Faiss et al., 2013; Gatterer et al.; CamachoCardenosa et al.).

In summary, based on the presented results of previous studies and known the underlying mechanisms that occur in hypoxic conditions, it could be concluded that RSH is an effective training method in improving physical performance among individual and team sport athletes. However, it should be noted that greater improvements were observed mainly in RSA tests compared to RSN, and less improvement was observed in aerobic YYIR tests. These results are line with the previous systematic reviews and meta-analyses (Brocherie et al., 2017; Millet et al.).

It is important to emphasize that the observed findings are more methodological in nature, because it is still unclear which methods to choose in terms of duration of stay in hypoxic conditions, exercise modalities (treadmill, pedaling, sprint), exercise volume and intensity, and especially recovery between sets and repetitions. Brocherie et al. (2017), suggested in their meta-analysis that the use of active rest in hypoxic conditions might be an inappropriate method because it slows down muscle re-oxygenation due to lower oxygen replacement in myoglobin and hemoglobin and reduced phosphocreatine resynthesis rate, which can lead to premature fatigue (Dupont et al., 2003; Faiss et al., 2013). Furthermore, it is good to notice that a large number of modalities of RSA tests might be leaded to the appearance of heterogeneity of results, which significantly complicates comparisons between the results. So, RSA tests should be unified, especially in terms of number of repetitions, duration or distance, as well as the duration and nature of rest for the reasons mentioned above. Also, given the specificity of training in hypoxic conditions and its effects, it would be desirable for future studies to monitor changes in the period after experimental treatments, in order to obtain information on how sustainable the effects are.

Finally, there were several strengths of this systematic review. Firstly, this systematic review was conducted in accordance with the PRISMA guidelines. Secondly, the study selection process was independently made by more than one researcher and exactly defined inclusion/exclusion criteria (minimized selection bias and increased internal validity). 
Thirdly, the comprehensively reported results (both text and tables) of this study were based on a large study population, both genders, different sport groups, and different ages, which was leading to more comprehensive review of the research topic. However, there were also some limitations, such as study designs of the selected studies varied, and there was used different training and test protocols, which might have impacted the physical performance or physiological adaptation. There could be also used a specific quality assessment tool (i.e. CONSORT checklist, Crowe Critical Appraisal Tool (CCAT) in this review to reduce the heterogeneity of the studies and further increase the generalizability of the results.

In the future it would be interesting to examine with randomized clinical trial study design the effects of RSH on physical performance and health both among individual and team sports athletes on-field in sport-specific settings and patients in the clinical settings. Additionally, the recommendation for further research, related to this research topic, would be to conduct a systematic review and metaanalysis to examine a larger number of high-quality of original studies with randomized clinical trial study design which would follow one type of training protocol, training conditions related to the fraction of inhaled air ( $\mathrm{FiO} 2)$, as well as performing sprints during training both on-field in sport-specific settings (i.e. among football players) or sprints on a bicycle ergometer in the laboratory environment (i.e. among cyclists).

\section{CONCLUSIONS}

In conclusion, based on current scientific studies, RSH is a promising and more effective method to improve the physical performance among individual and team sport athletes compared to repeated sprint training in normoxia (RSN). This systematic review study suggested that the RSH has a positive effect on the monitored variables in physical performance tests especially related to RSA.

ZELENOVIC, M.; KONTRO, T.; STOJANOVIC, T.; ALEXE, D. I.; BOZIC, D.; AKSOVIC, N.; BJELICA, B.; MILANOVIC, Z. \& ADRIAN, S. M. Efectos del entrenamiento de velocidad repetida en hipoxia sobre el rendimiento físico entre atletas: Una revisión sistemática. Int. J. Morphol., 39(6):1625-1634, 2021.

RESUMEN: El entrenamiento de velocidad repetida en hipoxia (RSH) representa un método innovador en el proceso de desarrollo y mejora del rendimiento físico entre los deportistas. Sin embargo, existen pocos datos científicos sobre este tema. El propósito de esta revisión sistemática fue investigar el efecto del método RSH sobre las habilidades motoras y el rendimiento de los atletas, obtener nueva información y ampliar las conclusiones ya conocidas. La búsqueda de datos se realizó en 4 bases de datos electrónicas: Google Scholar, PubMed, Web of Science e Research Gate para los años 2000- a mayo de 2021. Esta búsqueda se realizó en artículos en idioma inglés mediante el uso de los siguientes términos, individualmente / combinación: "capacidad de sprint repetido", "hipoxia", "efectos", "rendimiento físico" y " $\mathrm{VO}_{2 \max }$ " Se identificaron 844 estudios y se seleccionaron 14 de ellos (11 estudios realizados en hombres, un estudio realizado en mujeres y dos estudios realizados en ambos sexos). Los resultados mostraron, un tamaño muestral total de 347 atletas (40 mujeres y 307 hombres, de 15,3 \pm 0,5 - $35 \pm 7$ años). Se observó que la RSH fue un método de entrenamiento eficaz para mejorar todas las variables monitorizadas (es decir, RSAmax y $\mathrm{VO}_{2 \max }$ ). Sin embargo, se debe tener en consideración que se observaron mejoras importantes, principalmente, en las pruebas de capacidad de sprint repetido (RSA), y menos en las pruebas aeróbicas (es decir, Wingate y Yo-Yo). En conclusión, según los estudios científicos actuales, la RSH es un método más eficaz para mejorar el rendimiento físico entre los atletas en comparación con el entrenamiento de velocidad repetida en normoxia (RSN). Este estudio sugirió que la RSH tiene un efecto positivo sobre las variables monitoreadas en las pruebas de rendimiento físico especialmente relacionadas con RSA.

PALABRAS CLAVE: Capacidad de sprint repetido; Hipoxia; Efectos; Desempeño físico; $\mathrm{VO}_{2 \max }$

\section{REFERENCES}

Beard, A.; Ashby, J.; Chambers, R.; Brocherie, F.; Millet, G. P. RepeatedSprint Training in Hypoxia in International Rugby Union Players. Int J. Sports Physiol. Perform., 14(6):850-4, 2019.

Billaut, F.; Basset, F. A. \& Falgairette, G. Muscle coordination changes during intermittent cycling sprints. Neurosci. Lett., 380(3):265-9, 2005.

Billaut, F.; Kerris, J. P.; Rodriguez, R. F.; Martin, D. T.; Gore, C. J. \& Bishop, D. J. Interaction of central and peripheral factors during repeated sprints at different levels of arterial O2 saturation. PLoS One, 8(10):e77297, 2013.

Bishop, D.; Girard, O. \& Mendez-Villanueva, A. Repeated-sprint ability part II: recommendations for training. Sports Med., 41(9):741-56, 2011.

Bishop, D. J. \& Girard, O. Determinants of team-sport perfor-mance: implications for altitude training by team-sport athletes. Br. J. Sports Med., 47(Suppl 1):i17-21, 2013.

Bogdanis, G. C.; Nevill, M. E.; Boobis, L. H. \& Lakomy, H. K. Contribution of phosphocreatine and aerobic metabolism to energy supply during repeated sprint exercise. J. Appl. Physiol. (1985), 80(3):876-84, 1996.

Brechbuhl, C.; Brocherie, F.; Millet, G. P. \& Schmitt, L. Effects of repeatedsprint training in hypoxia on tennis-specific performance in well-trained players. Sports Med. Int. Open, 2(5):E123-32, 2018.

Brocherie, F.; Girard, O.; Faiss, R. \& Millet, G. P. Effects of repeatedsprint training in hypoxia on sea-level performance: A meta-analysis. Sports Med., 47(8):1651-60, 2017.

Brocherie, F.; Girard, O.; Faiss, R. \& Millet, G. P. High-intensity intermittent training in hypoxia: a double-blinded, placebo-controlled field study in youth football players. J. Strength Cond. Res., 29(1):226-37, 2015a.

Brocherie, F.; Millet, G. P.; Hauser, A.; Steiner, T.; Rysman, J.; Wehrlin, J. P. \& Girard, O. "Live high-train low and high" Hypoxic training improves team-sport performance. Med. Sci. Sports Exerc., 47(10):21409, 2015b. 
Buchheit, M.; Mendez-Villanueva, A.; Delhomel, G.; Brughelli, M. \& Ahmaidi, S. Improving repeated sprint ability in young elite soccer players: repeated shuttle sprints vs. explosive strength training. $J$. Strength Cond. Res., 24(10):2715-22, 2010.

Camacho-Cardenosa, A.; Camacho-Cardenosa, M.; Martinez-Guardado, I.; Brazo-Sayavera, J.; Timon, R.\& Olcina, G. Effects of repeated-sprint training in hypoxia on physical performance of team sports players. Rev. Bras. Med. Esporte, 26(2):153-57, 2020.

Casey, D. P. \& Joyner, M. J. Compensatory vasodilatation during hypoxic exercise: mechanisms responsible for matching oxygen supply to demand. J. Physiol., 590(24):6321-6, 2012.

Dekerle, J.; Mucci, P. \& Carter, H. Influence of moderate hypoxia on tolerance to high-intensity exercise. Eur. J. Appl. Physiol., 112(1):327-35, 2012.

Dupont, G.; Blondel, N. \% Berthoin S. Performance for short intermittent runs: active recovery vs. passive recovery. Eur. J. Appl. Physiol., 89(6):548-54, 2003.

Faiss, R.; Léger, B.; Vesin, J. M.; Fournier, P. E.; Eggel, Y.; Dériaz, O. \& Millet, G. P. Significant molecular and systemic adaptations after repeated sprint training in hypoxia. PLoS One., 8(2):e56522, 2013.

Faiss, R.; Willis, S.; Born, D. P.; Sperlich, B.; Vesin, J. M.; Holmberg, H. C. \& Millet, G. P. Repeated double-poling sprint training in hypoxia by competitive cross-country skiers. Med. Sci. Sports Exerc., 47(4):80917, 2015.

Fornasier-Santos, C.; Millet, G. P. \& Woorons X. Repeated-sprint training in hypoxia induced by voluntary hypoventilation improves running repeated-sprint ability in rugby players. Eur. J. Sport Sci., 18(4):504$12,2018$.

Galvin, H. M.; Cooke, K.; Sumners, D. P.; Mileva, K. N. \& Bowtell, J. L. Repeated sprint training in normobaric hypoxia. Br. J. Sports Med., 47 Suppl 1(Suppl 1):i74-i79, 2013.

Gatterer, H.; Philippe, M.; Menz, V.; Mosbach, F.; Faulhaber, M. \& Burtscher, M. Shuttle-run sprint training in hypoxia for youth elite soccer players: a pilot study. J. Sports Sci. Med., 13(4):731-5, 2014.

Girard, O.; Brocherie, F. \& Millet, G. P. On the use of mobile inflatable hypoxic marquees for sport-specific altitude training in team sports. Br. J. Sports Med., 47 Suppl 1(Suppl 1):i121-3, 2013.

Girard, O.; Mendez-Villanueva, A. \& Bishop, D. Repeated-sprint ability part I: factors contributing to fatigue. Sports Med., 41(8):673-94, 2011.

Gollnick, P. D.; Piehl, K. \& Saltin, B. Selective glycogen depletion pattern in human muscle fibres after exercise of varying intensity and at varying pedalling rates. J. Physiol., 241(1):45-57, 1974.

Goodall, S.; González-Alonso, J.; Ali, L.; Ross, E. Z. \& Romer L. M. Supraspinal fatigue after normoxic and hypoxic exercise in humans. $J$. Physiol., 590(11):2767-82, 2012.

Goods, P. S.; Dawson, B.; Landers, G. J.; Gore, C. J. \& Peeling, P. No additional benefit of repeat-sprint training in hypoxia than in normoxia on sea-level repeat-sprint ability. J. Sports Sci. Med., 14(3):681-8, 2015.

Goods, P. S. R.; Dawson, B. T.; Landers, G. J.; Gore, C. J. \& Peeling, P. Effect of different simulated altitudes on repeat-sprint performance in team-sport athletes. Int. J. Sports Physiol. Perform., 9(5):857-62, 2014.

Hamlin, M. J.; Olsen, P. D.; Marshall, H. C.; Lizamore, C. A. \& Elliot, C. A. Hypoxic repeat sprint training improves rugby player's repeated sprint but not endurance performance. Front. Physiol., 8:24, 2017.

Kasai, N.; Mizuno, S.; Ishimoto, S.; Sakamoto, E.; Maruta, M. \& Goto, K. Effect of training in hypoxia on repeated sprint performance in female athletes. Springerplus, 4:310, 2015.

Lundby, C. \& Robach, P. Does 'altitude training' increase exercise performance in elite athletes? Exp. Physiol., 101(7):783-8, 2016.

Millet, G. P.; Girard, O.; Beard, A. \& Brocherie, F. Repeated sprint training in hypoxia - an innovative method. Dtsch. Z. Sportmed., 5:115-22, 2019.

Moher, D.; Liberati, A.; Tetzlaff, J. \& Altman, D.G. \& for the PRISMA Group. Preferred reporting items for systematic reviews and metaanalyses: the PRISMA statement. BMJ, 339:b2535, 2009.

Pesta, D.; Hoppel, F.; Macek, C.; Messner, H.; Faulhaber, M.; Kobel, C.; Parson, W.; Burtscher, M.; Schocke, M. \& Gnaiger, E. Similar qualitative and quantitative changes of mitochondrial respiration following strength and endurance training in normoxia and hypoxia in sedentary humans. Am. J. Physiol. Regul. Integr. Comp. Physiol., 301(4):R1078-87, 2011.

Puype, J.; Van Proeyen, K.; Raymackers, J. M.; Deldicque, L. \& Hespel, P. Sprint interval training in hypoxia stimulates glycolytic enzyme activity. Med. Sci. Sports Exerc., 45(11):2166-74, 2013.

Rupp, T.; Leti, T.; Jubeau, M.; Millet, G. Y.; Bricout, V. A.; Levy, P.; Wuyam, B.; Perrey, S. \& Verges, S. Tissue deoxygenation kinetics induced by prolonged hypoxic exposure in healthy humans at rest. J. Biomed. Opt., 18(9):095002, 2013.

Serpiello, F. R.; McKenna, M. J.; Bishop, D. J.; Aughey, R. J.; Caldow, M. K.; Cameron-Smith, D. \& Stepto, N. K. Repeated sprints alter signaling related to mitochondrial biogenesis in humans. Med. Sci. Sports Exerc., 44(5):827-34, 2012.

Spencer, M.; Bishop, D.; Dawson, B. \& Goodman, C. Physiological and metabolic responses of repeated-sprint activities: specific to field-based team sports. Sports Med., 5(12):1025-44, 2005.

Westerblad, H.; Allen, D. G. \& Lännergren, J. Muscle fatigue: lactic acid or inorganic phosphate the major cause? News Physiol. Sci., 17:17-21, 2002.

\section{Corresponding author: \\ Milan Zelenovic \\ University of East Sarajevo \\ Faculty of Physical Education and Sport \\ Aleksa Santic no.3 \\ East Sarajevo 71123 \\ BOSNIA AND HERZEGOVINA}

E-mail: milanzeleni13@gmail.com

Received: 09-07-2021

Accepted: 25-08-2021 